\title{
ATIVIDADE MICROBIANA DE SOLO E SERAPILHEIRA EM ÁREAS POVOADAS COM Pinus elliottii E Terminalia ivorensis ${ }^{(1)}$
}

\author{
André Mundstock Xavier de Carvalho ${ }^{(2)}$, Helson Mário Martins do \\ Vale $^{(3)}$, Eráclides Maria Ferreira ${ }^{(4)}$, Alexander Francisco Perez \\ Cordero ${ }^{(5)}$, Nairam Félix de Barros ${ }^{(6)}$ \& Maurício Dutra Costa ${ }^{(7)}$
}

\section{RESUMO}

No Brasil, as espécies Pinus elliottii e Terminalia ivorensis vêm sendo indicadas para reflorestamento. No entanto, pouco se sabe sobre as características ecológicas destas florestas, o ciclo de nutrientes e suas conseqüências sobre a produtividade e sustentabilidade sob condições tropicais. Visando melhor compreender a dinâmica do C nestes ecossistemas, objetivouse neste trabalho avaliar a atividade microbiana do solo, serapilheira e da mistura solo + serapilheira em povoamentos florestais de $P$. elliottii e $T$. ivorensis. Amostras de solos e serapilheira foram incubadas e a atividade microbiana avaliada por meio da evolução de $\mathrm{CO}_{2}$. Ao final da incubação, a respiração acumulada foi superior para a serapilheira de $T$. ivorensis. Os demais substratos com serapilheira não diferiram entre si, mas diferiram do solo sob $T$. ivorensis, que, por sua vez, diferiu do solo sob $P$. elliottii. Nas condições testadas, a incorporação de solo à serapilheira, bem como a incorporação alternada de solo de um povoamento à serapilheira de outro, não promoveu aumentos significativos na respiração da serapilheira, mostrando que as características

\footnotetext{
(1) Trabalho apresentado no XXXI Congresso Brasileiro de Ciência do Solo, 2007 (Gramado, RS).

(2) Doutorando em Solos e Nutrição de Plantas da Universidade Federal de Viçosa - UFV. Departamento de Solos, Campus da UFV, s/n. Viçosa, MG, CEP 36570-000. E-mail: andremundstock@yahoo.com.br

(3) Doutorando em Microbiologia Agrícola. Departamento de Microbiologia (UFV). E-mail: valehmm@gmail.com.

(4) Doutoranda em Fitopatologia. Departamento de Fitopatologia (UFV). E-mail: ferreiraem@yahoo.com.br

(5) Professor da Universidade de Sucre - Colômbia. E-mail: alexpcor@yahoo.com.

(6) Professor titular do Departamento de Solos (UFV). E-mail: nfbarros@ufv.br.

(7) Professor adjunto do Departamento de Microbiologia (UFV). E-mail: mdcosta@ufv.br.
} 
químicas da própria serapilheira alteram mais fortemente sua velocidade de degradação que as características químicas e microbianas do solo onde é incorporada.

Termos de indexação: incorporação de serapilheira, degradação da serapilheira, respirometria.

\title{
SUMMARY: MICROBIAL ACTIVITY OF SOIL AND LITTER IN AREAS WITH FOREST STANDS OF PINUS ELLIOTTII E TERMINALIA IVORENSIS
}

\begin{abstract}
In Brazil, the species Pinus elliottii and Terminalia ivorensis are being recommended for reforestation. However, little is known about the ecological characteristics of such forests, the nutrient cycle and possible consequences on yields and sustainability under tropical conditions. For a better understanding of the $C$ dynamic in these ecosystems, this study aimed to evaluate the microbial activity of soil, litter, and the mixture of soil + litter in forest stands of $\mathrm{P}$. elliottii and T. ivorensis. Samples of soil, litter and mixture were incubated and the microbial activity was evaluated on the basis of $\mathrm{CO}_{2}$ released. At the end of incubation, the accumulated respiration was significantly higher in litter of T. ivorensis. The other substrates with litter did not differ from each other, but differed from the soil under T. ivorensis, which in turn differed from soil under P. elliottii. In the tested conditions, the soil incorporation to litter, as well as the alternate incorporation of soil from one forest with the litter of another did not cause a significant increase in litter respiration. This indicates that the influence of the chemical characteristics of the litter itself on degradation speed is stronger than the chemical and microbiological characteristics of the soil where it is incorporated.
\end{abstract}

Index terms: activity of the microbiota, litter incorporation, litter degradation.

\section{INTRODUÇÃO}

No Brasil, espécies de Pinus (Pinaceae) são plantadas com diversas finalidades, como produção de madeira para serraria, papel, chapas, aglomerados, dentre outras, sendo utilizadas de forma intensiva para reflorestamento (Caldeira et al., 1996; Iwakiri et al., 2002). Pinus elliottii está entre as espécies mais plantadas, mas relativamente pouco se conhece sobre as características ecológicas das florestas formadas com esta essência nas condições tropicais, principalmente em relação à cadeia alimentar e à ciclagem de nutrientes e suas conseqüências sobre a produtividade e sustentabilidade do sistema.

Terminalia ivorensis (Combretaceae) é uma espécie pioneira, decídua, de ocorrência natural na África equatorial, que se adapta bem em algumas regiões do Brasil e vem sendo indicada para reflorestamento de áreas desmatadas, graças, entre outros motivos, ao seu rápido crescimento. Sua madeira, comercialmente importante na África, pode ser usada para as mais diversas finalidades, principalmente para a carpintaria e, até mesmo, para a fabricação de papel (Baggio, 1997; Mattos et al., 1999). Existem, todavia, poucos estudos sobre esta espécie florestal (Urbinati et al., 2003), especialmente em referências nacionais (Mattos et al., 1999) e em estudos sobre dinâmica da serapilheira e ciclagem de nutrientes.

A ciclagem de nutrientes ocorre como conseqüência da atividade microbiana e é especialmente importante em ecossistemas de baixa fertilidade. Atividade dos microrganismos do solo pode ser avaliada de diversas formas, como pela medição da sua biomassa, da atividade de certas enzimas no solo, respiração microbiana basal (Tótola \& Chaer, 2002), dentre outras. Tais propriedades biológicas e bioquímicas do solo são consideradas indicadores sensíveis (Carvalho, 2005) que podem ser utilizados no monitoramento de alterações ambientais, constituindo-se, portanto, em ferramentas importantes para orientar o planejamento e a avaliação de práticas de manejo (Matsuoka et al., 2003). É sabido, no entanto, que os atributos bioquímicos que denotam processos edáficos dos ecossistemas, como a respiração microbiana, mostramse mais sensíveis para captar as alterações ocorridas no ambiente (Peña et al., 2005).

Assim como outros processos metabólicos, a respiração é dependente do estado fisiológico da célula 
e é influenciada por diversos fatores no solo, tais como: a umidade, a temperatura, a estrutura, a disponibilidade de nutrientes, a textura, a relação C/ $\mathrm{N}$, a presença de resíduos orgânicos, dentre outros (Carvalho, 2005).

Altas taxas de respiração podem significar, em curto prazo, liberação de nutrientes para as plantas e, em longo prazo, perda de C orgânico do solo para a atmosfera (Parkin et al., 1996). Nos solos tropicais, a matéria orgânica tem papel central na manutenção de sua fertilidade. No entanto, algumas práticas agrícolas e florestais, como o uso de corretivos e fertilizantes (Della Bruna et al., 1991), têm sido comumente associadas à redução do teor de matéria orgânica em razão, principalmente, do estímulo à respiração microbiana.

Dessa forma, visando melhor compreender a dinâmica do $\mathrm{C}$ nestes ecossistemas, objetivou-se neste trabalho avaliar a atividade microbiana do solo, serapilheira e da mistura solo + serapilheira em povoamentos florestais de $P$. elliottii e T. ivorensis.

\section{MATERIAL E MÉTODOS}

\section{Amostragem e preparo do material}

Os povoamentos de $P$. elliottii e $T$. ivorensis estudados estão localizados no município de
Viçosa, MG, Brasil (2046'18,7” S; 4252'37,6” W e $20^{\circ} 46$ '19,2" S $42^{\circ} 52^{\prime} 37,2$ " W, respectivamente), altitude de 668 m, classificação climática de Köppen - Cwb. Os povoamentos apresentam 36 anos de idade e o espaçamento entre plantas em ambos é de $2,5 \mathrm{x}$ $2,5 \mathrm{~m}$, perfazendo uma área de aproximadamente 0,06 ha cada.

As amostras foram coletadas na estação seca, mês de julho. Foram demarcados, aleatoriamente, oito pontos em cada área onde amostras simples de solo e serapilheira foram coletadas. Para amostragem do solo, realizada com pá de corte até $20 \mathrm{~cm}$, foi removida a camada de serapilheira. Para amostragem da serapilheira, utilizou-se um gabarito de $0,04 \mathrm{~m}^{2}$, sendo o material armazenado em sacos plásticos. A massa das amostras de serapilheira fresca e a seca, a umidade e a capacidade de retenção de água dos solos e das serapilheiras foram determinadas. Uma segunda coleta de amostras, três dias após a primeira, foi feita igualmente nos pontos demarcados e armazenada individualmente para aquisição de material em quantidade suficiente para montagem do experimento. As amostras de solo foram peneiradas (malha de $2 \mathrm{~mm}$ ) e as amostras de serapilheira foram homogeneizadas e picadas em fragmentos inferiores a $4 \mathrm{~cm}$ de comprimento. Os materiais foram imediatamente incubados para determinação da atividade microbiana. Uma amostra composta de cada um dos materiais foi submetida a análises físicas e químicas (Quadro 1).

Quadro 1. Características físico-químicas ${ }^{(1)}$ do solo e da serapilheira dos povoamentos de Terminalia ivorensis e Pinus elliottii

\begin{tabular}{|c|c|c|c|c|c|}
\hline Solo & T. ivoren. & P. elliotti & Serapilheira & T. ivoren. & P. elliotti \\
\hline $\mathrm{pH}_{\mathrm{H} 2 \mathrm{O}}$ & 5,5 & 4,8 & $\mathrm{~N}\left(\right.$ dag kg$\left.^{-1}\right)$ & 1,13 & 0,65 \\
\hline $\mathrm{P}\left(\mathrm{mg} \mathrm{dm}^{-3}\right)$ & 1,1 & 1,2 & $\mathrm{P}($ dag kg-1) & 0,04 & 0,03 \\
\hline $\mathrm{K}\left(\mathrm{mg} \mathrm{dm}^{-3}\right)$ & 48 & 27 & $\mathrm{~K}\left(\mathrm{dag} \mathrm{kg}^{-1}\right)$ & 0,24 & 0,18 \\
\hline $\mathrm{Ca}^{2+}\left(\mathrm{cmol}_{\mathrm{c}} \mathrm{dm}^{-3}\right)$ & 4,01 & 1,58 & $\mathrm{Ca}^{2+}\left(\right.$ dag kg $\left.{ }^{-1}\right)$ & 3,09 & 0,64 \\
\hline $\mathrm{Mg}^{2+}\left(\mathrm{cmol}_{\mathrm{c}} \mathrm{dm}^{-3}\right)$ & 1,17 & 0,45 & $\mathrm{Mg}^{2+}\left(\mathrm{dag} \mathrm{kg}^{-1}\right)$ & 0,20 & 0,11 \\
\hline $\mathrm{Al}^{3+}\left(\mathrm{cmol}_{\mathrm{c}} \mathrm{dm}^{-3}\right)$ & 0,00 & 0,15 & $\mathrm{~S}\left(\mathrm{mg} \mathrm{kg}^{-1}\right)$ & 0,07 & 0,04 \\
\hline $\mathrm{H}+\mathrm{Al}\left(\mathrm{cmol}_{\mathrm{c}} \mathrm{dm}^{-3}\right)$ & 2,70 & 4,80 & $\mathrm{Zn}\left(\mathrm{mg} \mathrm{kg}^{-1}\right)$ & 25,60 & 16,20 \\
\hline $\mathrm{SB}\left(\mathrm{cmol}_{\mathrm{c}} \mathrm{dm}^{-3}\right)$ & 5,30 & 2,10 & $\mathrm{Fe}\left(\mathrm{mg} \mathrm{kg}^{-1}\right)$ & 3846,50 & 449,80 \\
\hline CTC efetiva $\left(\mathrm{cmol}_{\mathrm{c}} \mathrm{dm}^{-3}\right)$ & 5,30 & 2,25 & $\operatorname{Mn}\left(\mathrm{mg} \mathrm{kg}^{-1}\right)$ & 260,00 & 421,40 \\
\hline $\mathrm{CTC}_{\mathrm{pH} 7,0}\left(\mathrm{cmol}_{\mathrm{c}} \mathrm{dm}^{-3}\right)$ & 8,00 & 6,90 & $\mathrm{Cu}\left(\mathrm{mg} \mathrm{kg} \mathrm{g}^{-1}\right)$ & 15,50 & 3,50 \\
\hline $\mathrm{V}(\%)$ & 66,3 & 30,4 & & & \\
\hline $\mathrm{m}(\%)$ & 0,0 & 6,7 & & & \\
\hline MO (dag kg-1) & 3,01 & 2,76 & & & \\
\hline P-rem (mg L-1) & 39,4 & 37,9 & & & \\
\hline Umidade (\%) & 12,5 & 15,7 & Umidade (\%) & 33,97 & 52,55 \\
\hline Retenção de água (\%) & 19 & 17 & Retenção de água (\%) & 100 & 100 \\
\hline
\end{tabular}

${ }^{(1)}$ Análises químicas foram realizadas no Laboratório de Análises Químicas de Solo do Departamento de Solos da Universidade Federal de Viçosa, segundo método descrito pela Empresa Brasileira de Pesquisa Agropecuária-Embrapa (1997). 


\section{Atividade microbiana do solo}

A atividade microbiana do solo foi avaliada pela respiração, indiretamente determinada pela evolução de $\mathrm{CO}_{2}$, conforme método descrito por Alef \& Nannipieri (1995).

Utilizou-se o delineamento inteiramente casualizado com os tratamentos arranjados em parcelas subdivididas no tempo ( $8 \times 10)$, sendo oito tipos de substratos, em dez tempos de incubação, com 4 repetições. As combinações de substratos foram: S0 - controle (sem substrato), S1 solo P. elliottii, S2 - serapilheira P. elliottii, S3 - solo P. elliottii + serapilheira P. elliottii, S4 - solo P. elliottii+ serapilheira T. ivorensis, S5 - solo T. ivorensis, S6 serapilheira T. ivorensis, S7 - solo T. ivorensis + serapilheira T. ivorensis, S8 - solo T. ivorensis + serapilheira $P$. elliottii. A liberação de $\mathrm{CO}_{2}$ foi avaliada nos tempos 12, 21, 40, 64, 89, 131, 176, 225, 321 e $439 \mathrm{~h}$ de incubação. Para isso, frascos de vidro com volume igual a $800 \mathrm{mLque}$ continham as diferentes combinações de substratos e béqueres com $20 \mathrm{~mL}$ de $\mathrm{NaOH}$ 0,5 mol $\mathrm{L}^{-1}$ foram hermeticamente fechados e incubados a 25 ${ }^{\circ} \mathrm{C}$, no escuro. A massa de substrato/frasco foi o correspondente, em peso do material seco, a $150 \mathrm{~g}$ de solo (S1 e S5), 25 g de serapilheira (S2 e S6) e $150 \mathrm{~g}$ de solo +25 g de serapilheira (S3, S4, S7 e S8). Em cada tempo de avaliação, os frascos foram abertos e a solução de $\mathrm{NaOH}$ trocada. Imediatamente após a retirada dos béqueres foi adicionado $1 \mathrm{~mL}$ da solução de $\mathrm{BaCl}_{2} 50 \%$ e acrescentadas três gotas do indicador fenolftaleína $1 \%$. O NaOH restante, não precipitado como $\mathrm{Na}_{2} \mathrm{CO}_{3}$, foi titulado com $\mathrm{HCl} 0,5 \mathrm{~mol} \mathrm{~L}^{-1}$. A partir do volume de $\mathrm{HCl}$ consumido, foi calculada a quantidade de $\mathrm{C}-\mathrm{CO}_{2}$ liberado por grama de carbono do substrato seco. Previamente, a umidade dos solos utilizados na incubação foi corrigida para $30 \%$ (aproximadamente 60 $\%$ da capacidade de retenção de ambos os solos). A umidade da serapilheira não foi alterada permanecendo próxima a $75 \%$ (Quadro 1).

\section{Análises estatísticas}

As análises estatísticas foram processadas com auxílio dos programas Saeg 9.1 (Euclydes, 1983) e SigmaPlot 10.0. (SPSS, 2006). Os dados foram submetidos à análise de variância e à análise de regressão ( $\mathrm{mg} \mathrm{g}^{-1}$ de $\mathrm{C}-\mathrm{CO}_{2}$ do $\mathrm{C}$ do substrato seco de acordo com o tempo de incubação). Para comparação entre médias, utilizaram-se o teste $\mathrm{F}$ (acúmulo de serapilheira) e o teste de Tukey (respiração acumulada e taxa respiratória). Para comparações entre grupos de tratamentos, foram estabelecidos contrastes que foram testados pelo teste de Scheffè.

\section{RESULTADOS}

Os povoamentos apresentaram diferenças significativas nas massas de serapilheira (seca e fresca), sendo maiores no povoamento de P. elliottii (Figura 1).

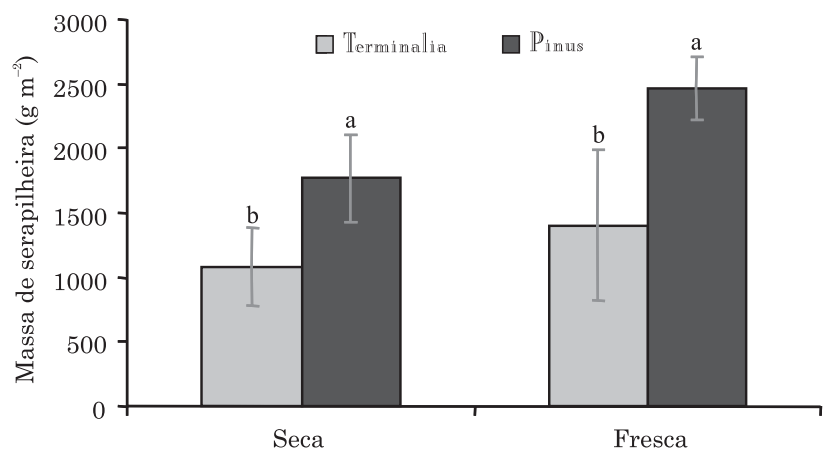

Figura 1. Massa da serapilheira seca e fresca acumulada nos povoamentos de Terminalia ivorensis e Pinus elliottii. Barras seguidas por uma mesma letra não diferem estatisticamente entre si pelo teste $\mathrm{F}(\mathrm{p}<\mathbf{0 , 0 3})$. Os desvios estão representados por barras.

Em todos os substratos, foi observada uma redução na taxa respiratória com o tempo. O comportamento desta taxa, no entanto, diferiu entre os substratos que continham serapilheira e os que continham apenas solo, como demonstrado pelos modelos de regressão ajustados nos dois casos (Figura 2).

A interação entre os fatores substrato e tempo de incubação foi significativa $(p<0,05)$ tanto para as taxas respiratórias como para a respiração acumulada. Na presença da serapilheira (S2, S3, $\mathrm{S} 4, \mathrm{~S} 6, \mathrm{~S} 7, \mathrm{~S} 8$ ), as maiores taxas de respiração ocorreram nas primeiras $12 \mathrm{~h}$ de incubação (Figura 2a). Nas horas seguintes, as taxas respiratórias caíram acentuadamente, voltando a aumentar próximo às $64 \mathrm{~h}$ de incubação. Após este novo incremento, finalmente, as taxas respiratórias reduziram-se gradualmente até o final da incubação, indicando a estabilização da atividade microbiana (Figura 2).

Nos solos sob T. ivorensis e P. elliottii (S5 e S1), por outro lado, houve apenas rápida elevação nas taxas respiratórias às $21 \mathrm{~h}$ de incubação, quando atingiram um máximo em valores de 0,21 e $0,08 \mathrm{mg} \mathrm{g}^{-1} \mathrm{~h}^{-1} \mathrm{de} C$ $\mathrm{CO}_{2}$ do $\mathrm{C}$ do substrato seco, respectivamente. Logo após, iniciou-se a redução gradual das taxas até à estabilização em aproximadamente $0,04 \mathrm{mg} \mathrm{g}^{-1} \mathrm{~h}^{-1}$ de C- $\mathrm{CO}_{2}$ do $\mathrm{C}$ do substrato seco $\mathrm{h}^{-1}$ para ambos os solos (Figura 2).

Nas primeiras $64 \mathrm{~h}$ de incubação, menores taxas respiratórias foram observadas nos substratos que continham serapilheira de $P$. elliottii em relação aos de T. ivorensis pelo teste de Scheffè $(p<0,01)$. Os substratos com serapilheira apresentaram maiores taxas respiratórias que aqueles que continham apenas solo até $225 \mathrm{~h}$ de incubação, igualando-se ao final da incubação $(p<0,05)$. O solo sob $T$. ivorensis apresentou maior taxa respiratória que o solo sob $P$. elliottii apenas nas primeiras $21 \mathrm{~h}$ de incubação $(\mathrm{p}<0,05)$. 

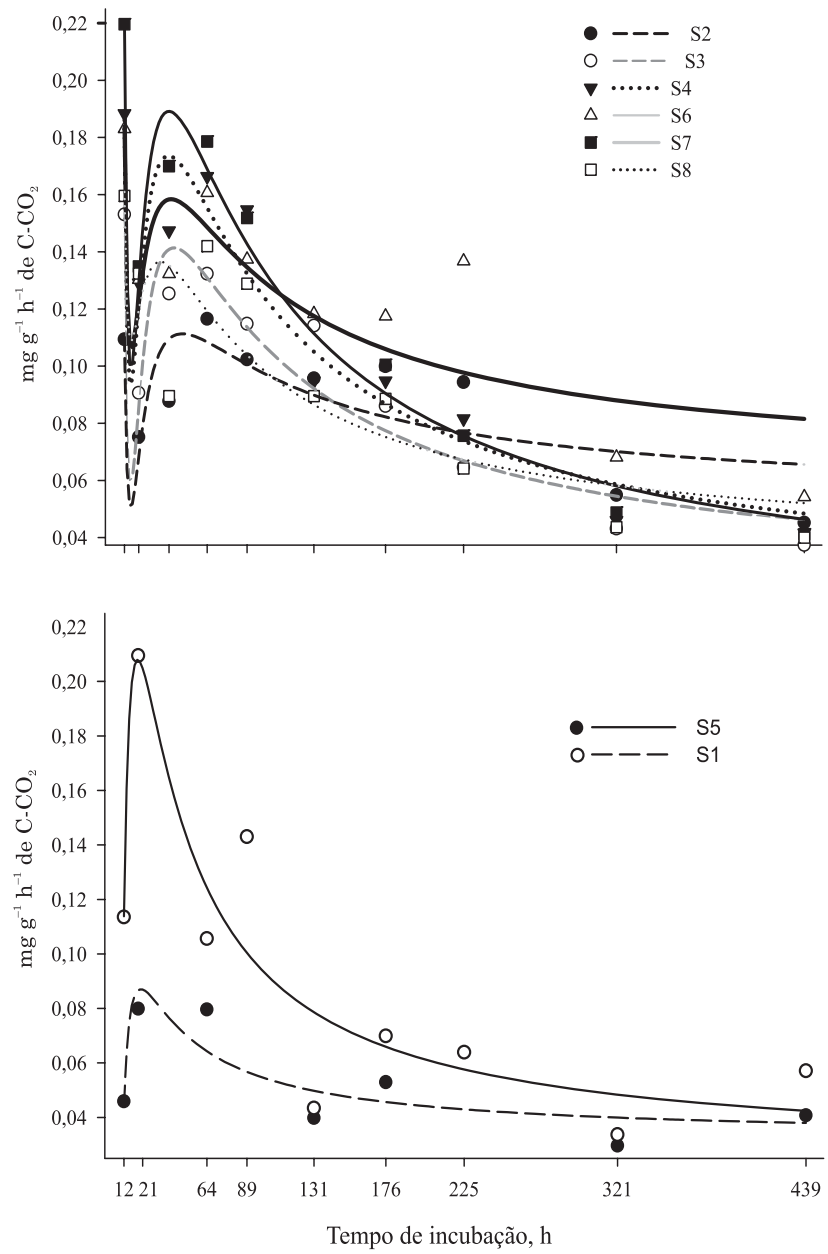

Figura 2. Taxas respiratórias de serapilheira, solo e diferentes combinações serapilheira/solo de Pinus elliottii e Terminalia ivorensis. S1 - solo/ $P$. elliottii, S2 - serapilheira/P. elliottii, S3 - solo/ P. elliottii + serapilheira/P. elliottii, $\mathbf{S} 4-$ solo/ P. elliottii + serapilheira/T. ivorensis, S5 - solo/ T. ivorensis, $\mathrm{S} 6$ - serapilheira/T. ivorensis, $\mathrm{S} 7$ solo/T. ivorensis + serapilheira/T. ivorensis, $\mathrm{S} 8$ - solo/T. ivorensis + serapilheira/P. elliottii. * ,

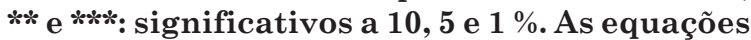
ajustadas foram:

$\hat{\mathbf{s}}_{1}=0,0325+2,4685 * * / \mathrm{x}-27,9466^{* *} / \mathrm{x}^{2}\left(\mathrm{R}^{2}=0,765\right)$;

$\hat{\mathrm{s}}_{2}=0,0519+6,4460 * * / \mathrm{x}-207,2294 * * / \mathrm{x}^{2}+$ $1659,0872^{* * / x^{3}}\left(R^{2}=0,605\right)$;

$\hat{\mathrm{S}}_{3}=11,8627 * * * / \mathrm{x}-350,6417 * * * / \mathrm{x}^{2}+2728,9680^{* * *} / \mathrm{x}^{3}$ $\left(\mathrm{R}^{2}=0,922\right)$

$\hat{\mathbf{S}}_{4}=0,0179+14,2691 * * * / \mathrm{x}-396,9054 * * * / \mathrm{x}^{2}+$ $3003,9880 * * * / \mathrm{x}^{3}\left(\mathrm{R}^{2}=0,921\right)$

$\hat{\mathrm{S}}_{5}=0,0257+7,5161^{* * *} / \mathrm{x}-77,5039 * * * / \mathrm{x}^{2}\left(\mathrm{R}^{2}=0,846\right) ;$

$\hat{\mathbf{S}}^{6}=0,0620+9,1703 * * / \mathrm{x}-264,1884 * * / \mathrm{x}^{2}+$ $2060,4242^{* *} / \mathrm{x}^{3}\left(\mathrm{R}^{2}=0,722\right)$;

$\hat{\mathrm{s}}_{7}=0,0113+16,4366 * * * / \mathrm{x}-461,0077 * * * / \mathrm{x}^{2}+$ $3526,2292^{* * *} / \mathrm{x}^{3}\left(\mathrm{R}^{2}=0,974\right)$;

$\hat{\mathbf{s}}_{8}=0,0339+8,4298 * * / \mathrm{x}-214,5319 * / \mathrm{x}^{2}+1579,9453 * /$ $\mathrm{x}^{3}\left(\mathrm{R}^{2}=0,745\right)$.
A respiração acumulada ao longo do período de incubação tendeu à estabilização em todos os substratos, com exceção do solo sob $P$. elliottii, onde o modelo quadrático de regressão não foi apropriado (Figura 3).

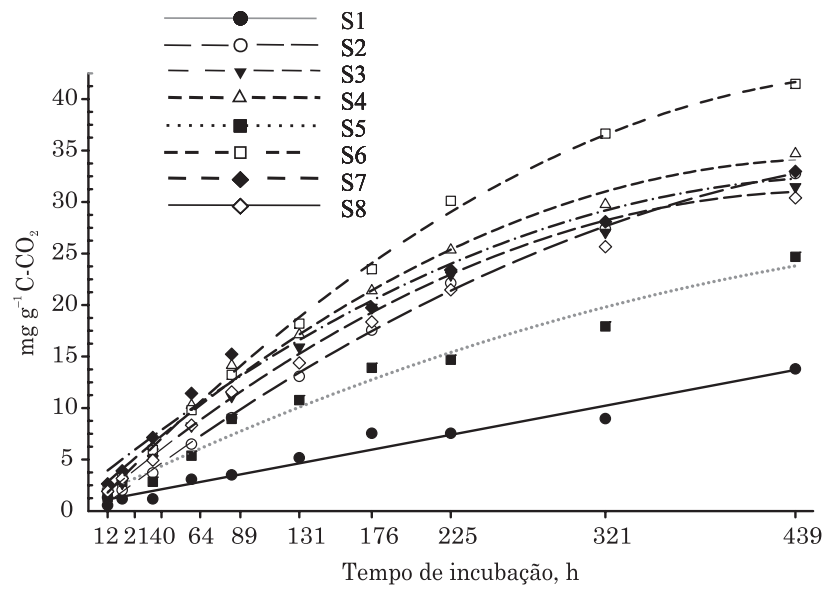

Figura 3. Respiração acumulada $\left(\mathrm{mg} \mathrm{g}^{-1} \mathrm{de} \mathrm{C}^{-\mathrm{CO}_{2}}\right.$ de C do substrato seco) ao longo do período de incubação de serapilheira, solo e diferentes combinações serapilheira/solo de Pinus elliottii e Terminalia ivorensis. S1 - solo/P. elliottii, S2 serapilheira/P. elliottii, S3 - solo/P. elliottii + serapilheira/P. elliottii, S4 - solo/P. elliottii + serapilheira/T. ivorensis, S5 - solo/T. ivorensis, S6 - serapilheira/T. ivorensis, S7 - solo/T. ivorensis + serapilheira/T. ivorensis, $\mathrm{S} 8$ - solo/T. ivorensis + serapilheira/P. elliottii. ** e *: significativos a 1 e $5 \%$. As equações ajustadas foram: $\hat{\mathbf{s}}_{1}=24,0997 *\left(1-0,9982^{* * x}\right)\left(\mathrm{R}^{2}=0,985\right)$; $\hat{\mathrm{s}}_{2}=53,5745^{* *}\left(1-0,9978^{* * \mathrm{x}}\right)\left(\mathrm{R}^{2}=0,998\right)$; $\hat{\mathrm{S}}_{3}=37,9361^{* *}\left(1-0,9960^{* * \mathrm{x}}\right)\left(\mathrm{R}^{2}=0,999\right)$; $\hat{\mathrm{s}}_{4}=39,8319^{* *}\left(1-0,9955^{* * \mathrm{x}}\right)\left(\mathrm{R}^{2}=0,999\right)$; $\hat{\mathrm{S}}_{5}=32,0087^{* *}\left(1-0,9970^{* * \mathrm{x}}\right)\left(\mathrm{R}^{2}=0,989\right)$; $\hat{\mathbf{s}}_{6}=57,3274^{* *}(1-0,9969 * * x)\left(\mathrm{R}^{2}=0,998\right)$; $\hat{\mathbf{s}}_{7}=34,7983^{* *}\left(1-0,9946^{* * x}\right)\left(\mathrm{R}^{2}=0,993\right)$; $\hat{\mathrm{S}}_{8}=36,3865^{* *}\left(1-0,9960^{* * \mathrm{x}}\right)\left(\mathrm{R}^{2}=0,999\right)$.

A respiração acumulada dos substratos com serapilheira de $T$. ivorensis foi superior à dos com serapilheira de $P$. elliottii somente a partir da $225^{\mathrm{a}} \mathrm{h}$ de incubação $(p<0,05)$. Por outro lado, a serapilheira diferiu do solo quanto à respiração acumulada já a partir de $89 \mathrm{~h}$ de incubação $(\mathrm{p}<0,05)$. Ao final da incubação a respiração acumulada foi significativamente superior para a serapilheira de T. ivorensis, em 26,6, 31,7 e 36,4 \%, quando comparada à dos substratos $\mathrm{S} 2$, S3 e S8, respectivamente. Os demais substratos com serapilheira não diferiram entre si, mas diferiram do solo sob T. ivorensis que, por sua vez, diferiu do solo sob $P$. elliottii pelo teste Tukey $(\mathrm{p}<0,01)($ Figura 4). 
Os dados evidenciaram que, nas condições testadas, a incorporação de serapilheira ao solo promoveu aumentos significativos na respiração do solo, mas não na respiração da serapilheira. No solo de $P$. elliottii (S1), por exemplo, a liberação total de $\mathrm{CO}_{2}$ foi aumentada em 151,7, 139,2, 128,4 e 120,5 \% na presença de serapilheira (S4, S7, S3 e S8, respectivamente). Já a incorporação alternada de solo de um povoamento com a serapilheira de outro não alterou a taxa respiratória da serapilheira em relação aos substratos não alternados.

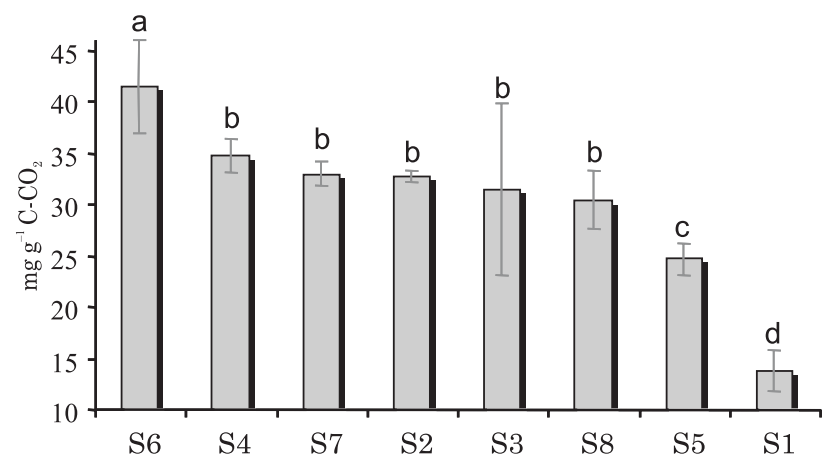

Figura 4. Respiração acumulada ao final do período de incubação de serapilheira, solo e diferentes combinações serapilheira/solo de Pinus elliottii e Terminalia ivorensis. S1 - solo/P. elliottii, $\mathrm{S} 2-$ serapilheira/P. elliottii, S3 - solo/P. elliottii + serapilheira/P. elliottii, S4 - solo/P. elliottii + serapilheira/T. ivorensis, S5 - solo/T. ivorensis, S6 - serapilheira/T. ivorensis, $\mathrm{S} 7$ - solo/T. ivorensis + serapilheira/T. ivorensis, $\mathrm{S} 8$ - solo/T. ivorensis + serapilheira/P. elliottii. Colunas seguidas pela mesma letra não diferem, entre si, pelo teste de Tukey $(\mathrm{p}<0,01)$. Os desvios estão representados por barras.

\section{DISCUSSÃO}

No povoamento de $P$. elliottii, houve maior acúmulo de serapilheira em relação ao povoamento de T. ivorensis (Figura 1), o que pode estar diretamente relacionado com o menor teor de nutrientes para todos os elementos analisados neste material, exceto o Mn (Quadro 1), ou, ainda, com os prováveis maiores teores de compostos fenólicos e ligninas ou maior relação $\mathrm{C} / \mathrm{N}$ nas acículas. Esses fatores podem alterar direta e negativamente o crescimento e a disponibilidade do $\mathrm{C}$ à ação da microbiota (Monteiro \& Gama-Rodrigues, 2004; Gama-Rodrigues et al., 2005). Além disso, o solo sob este povoamento apresentou, de modo geral, menores índices de fertilidade, o que pode ter acentuado ainda mais a carência de nutrientes para a microbiota.

Rigobelo \& Nahas (2004), estudando a atividade microbiana em solos de Eucalyptus e Pinus, verificaram que maior atividade microbiana ocorreu no solo com maior teor de nutrientes. No entanto, a deposição de serapilheira em coníferas, como o Pinus, mostrou-se comumente inferior à de espécies arbóreas folhosas (Florence \& Lamb 1974; Rigobelo \& Nahas 2004).

O acúmulo de serapilheira foi mediado pela quantidade de material que cai da parte aérea das plantas e por sua velocidade de decomposição (Heal et al., 1997). Os substratos que continham serapilheira de $P$. elliottii apresentaram, de modo geral, valores acumulados de respiração inferiores aos da serapilheira de T. ivorensis, o que significa menor atividade microbiana e menor degradação da matéria orgânica sobre o solo (Figura 3). Do mesmo modo, as taxas de evolução de $\mathrm{CO}_{2}$ destes substratos diferiram nas primeiras horas de incubação, quando a velocidade de produção foi maior nos substratos com serapilheira de T. ivorensis pelo teste de Scheffè $(p<0,01)$.

Assim, o maior acúmulo de serapilheira no povoamento de $P$. elliottii pode estar mais fortemente ligado à menor atividade microbiana neste material (Figura 4), por motivos já discutidos, o que deve ter compensado uma possível menor deposição de litter. $\mathrm{E}$, finalmente, a maior deposição de litter no povoamento de T. ivorensis pode estar ligada à menor umidade do solo sob $P$. elliottii e à época de amostragem, uma vez que, como decídua, as plantas de T. ivorensis podem ter elevado momentaneamente a deposição de folhas.

As quedas nas taxas respiratórias para todos os substratos (Figura 2) podem ser explicadas pelo consumo gradual das fontes de $\mathrm{C}$ mais prontamente mineralizáveis. Nos substratos com apenas solo (Figura 2), menores taxas foram encontradas a partir de $89 \mathrm{~h}$ de incubação provavelmente em virtude da qualidade do $\mathrm{C}$ e não de seus teores, uma vez que a taxa foi expressa em $\mathrm{mg}$ de $\mathrm{C}-\mathrm{CO}_{2}$ liberado por grama de carbono do substrato. Na matéria orgânica do solo, espera-se encontrar formas quimicamente mais complexas do $\mathrm{C}$ que na serapilheira, o que desfavorece a utilização do $\mathrm{C}$ pela microbiota. Uma alta taxa respiratória indicará maior atividade microbiana e uma decomposição mais rápida do material orgânico do solo, com conseqüente liberação mais elevada de nutrientes para as plantas (Carter, 1986).

Os picos nas taxas respiratórias apresentados por todos os substratos com serapilheira (Figura 2) podem ser atribuídos ao esgotamento seqüencial das diferentes fontes de $\mathrm{C}$, onde aquelas de menor complexidade estrutural e melhor equilíbrio de nutrientes foram primeiramente e rapidamente consumidas (até $21 \mathrm{~h}$ ). Quanto menor for a relação C/ $\mathrm{N}$, por exemplo, mais rápida é a liberação de $\mathrm{CO}_{2}$ (Araújo et al., 1999).

A queda na taxa respiratória, observada às $21 \mathrm{~h}$ de incubação, para os substratos com serapilheira, pode estar ligada, portanto, ao esgotamento das fontes mais prontamente mineralizáveis, a partir do qual a 
microbiota necessitou de tempo para se adaptar fisiologicamente à utilização de fontes mais complexas (Tauk, 1990; Moat et al., 2002), mas ainda de considerável disponibilidade, atingindo um novo pico próximo às $64 \mathrm{~h}$ de incubação. Após o esgotamento destas, por sua vez, as taxas voltaram a cair, evidenciando ter restado apenas fontes de baixa disponibilidade e estabilização da atividade microbiana.

A diferença encontrada na respiração acumulada entre os dois solos (Figura 3) pode estar relacionada com a origem da matéria orgânica e com a melhor fertilidade do solo observada sob povoamento de $T$. ivorensis, visto que a quantidade de nutrientes interfere na atividade microbiana (Assis Júnior et al., 2003; Monteiro \& Gama-Rodrigues, 2004).

Embora não tenham ocorrido diferenças (Figura 4), a mistura da serapilheira com solo sabidamente influencia a ciclagem de nutrientes e é importante para a microfauna do solo. Fungos e actinomicetos, por exemplo, decompõem materiais mais resistentes, tais como celulose, hemicelulose e lignina. Besouros, minhocas e outros decompositores aumentam as taxas de decomposição de fezes e serapilheira pela mistura deles com o solo (González et al., 2001). Assim, este resultado quanto ao efeito da mistura da serapilheira ao solo talvez não corresponda às condições de campo.

Della Bruna et al. (1991) também demonstraram que a adição de serapilheira ao solo pode aumentar a atividade biológica nos solos em condições de laboratório sem, no entanto, distinguir o efeito devido ao incremento no teor de $\mathrm{C}$ ao solo pela adição da serapilheira. Sabe-se que a incorporação de materiais como serapilheira pode proporcionar aumento ou manutenção da produtividade de determinada área. Os processos envolvidos estão relacionados com atividades que maximizam a produção, como a entrada de nutrientes via atividade microbiana. Esses processos devem ser mais bem estudados para melhoria de produção de plantas comercialmente importantes.

\section{CONCLUSÕES}

1. A atividade da microbiota foi maior nos substratos com serapilheira de T. ivorensis que nos de serapilheira de $P$. elliottii, sendo este o principal motivo que explica o maior acúmulo de serapilheira observado no povoamento de $P$. elliottii.

2. A atividade da microbiota foi superior nos substratos com serapilheira, seguido pelo solo de $T$. ivorensis e, finalmente, pelo de $P$. elliottii em razão, provavelmente, da disponibilidade de nutrientes e da qualidade biológica do $\mathrm{C}$ presente nestes materiais.

3. A incorporação de solo à serapilheira, bem como a incorporação alternada de solo de um povoamento com a serapilheira de outro, não promoveu aumentos significativos na respiração da serapilheira, evidenciando que, nas condições de incubação, as características químicas da própria serapilheira determinam mais fortemente a velocidade de degradação que as características químicas e microbianas do solo onde é incorporada.

\section{AGRADECIMENTOS}

Ao Conselho Nacional de Desenvolvimento Científico e Tecnológico (CNPq).

\section{LITERATURA CITADA}

ALEF, K. \& NANNIPIERI, D. Methods in applied soil microbiology and biochemistry. London, Academic Press, 1995. p.214-216.

ARAÚJO, T.M.; CARVALHO Jr., J.A.; HIGUCHI, N.; BRASIL, Jr., A.C.P. \& MESQUITA, A.L.A. A tropical rain forest clearing experiment by biomass burning in the State of Pará, Brazil. Atmos. Environ., 33:1991-1998, 1999.

ASSIS JUNIOR, S.L.; ZANUNCIO, J.C.; KASUYA, M.C.M.; COUTO, L. \& MELIDO, R.C.N. Soil microbial activity in agroforest, monocultures, natural forest and deforested area systems. R. Árvore, 27:35-41, 2003.

BAGGIO, J.A. Introdução de Terminalia ivorensis em CIANORTE, PR. B. Pesq. Flor., 35:85-90, 1997.

CALDEIRA, M.C.W.; TONINI, H.; HOPPE, J.M.; WATZLAWICK, L.F. \& SELLE, G.L. Definição de sítios em povoamentos de Pinus elliottii Engelm. na região de encruzilhada do sul - RS. Ci. Flor., 6:1-13, 1996.

CARTER, M.R. Microbial biomass as an index for tillage induced changes in soil biological properties. Soil Till Res., 7:29-40, 1986.

CARVALHO, F. Atributos bioquímicos como indicadores da qualidade de solo em floresta de Araucaria angustifolia (Bert.) O. Ktze no Estado de São Paulo. Piracicaba, Escola Superior de Agricultura Luiz de Queiroz, 2005. 79p. (Tese de Mestrado)

DELLA BRUNA, E.; BORGES, A.C.; FERNANDES, B.; BARROS, N.F. \& MUCHOVEJ, R.M.C. Atividade da microbiota de solos adicionados de serapilheira de eucalipto e de nutrientes. R. Bras.Ci. Solo, 15:15-20, 1991.

EMPRESA BRASILEIRA DE PESQUISA AGROPECUÁRIA EMBRAPA. Centro Nacional de Pesquisa de Solos. Manual de métodos de análise de solo. 2.ed. Rio de Janeiro, 1997. 212p.

EUCLYDES, R.F. Sistema de análises estatísticas e genéticas - SAEG. Viçosa, MG, Universidade Federal de Viçosa, 1983. 68p.

FLORENCE, R.O. \& LAMB, D. Influence of stand and site on radiata pine litter in South Austra1ia. New Zealand J. For. Sci., 4:502-10, 1974. 
GAMA-RODRIGUES, E.F.; BARROS, N.F.; GAMARODRIGUES, A.C. \& SANTOS, G.A. Carbon, nitrogen and activity of microbial biomass in soil under eucalypt plantations. R. Bras. Ci. Solo, 29:893-901, 2005.

GONZÁLEZ, G.; LEY, R.E.; SCHMIDT, S.K.; ZOU, X. \& SEASTEDT, T.R. Soil ecological interactions: Comparisons between tropical and subalpine tropics. Oecologia, 128:549-556, 2001.

HEAL, O.W.; ANDERSON, F.M. \& SWIFT, M.F. Plant litter quality and decomposition: An historical overview. In CADISCH, G. \& GILLER, K.E., eds. Cambridge, CAB International, 1997. p.3-32.

IWAKIRI, S.; SILVA, J.C.; SILVA, J.R.M.; ALVES, C.R. \& PUEHING, C.A. Plywood manufacturing from Pinus elliottii L. and Pinus oocarpa Schiede with different formulations of the urea-formaldheyde resin. R. Árvore, 26:371-375, 2002.

MATSUOKA, M.; MENDES, I.C. \& LOUREIRO, M.F. Biomassa microbiana e atividade enzimática em solos sob vegetação nativa e sistemas agrícolas anuais e perenes na região de Primavera do Leste (MT). R. Bras. Ci. Solo, 27:425-433, 2003.

MATTOS, P.P.; PEREIRA, J.C.D.; SCHAITZA, E.G. \& BAGGIO, A.J. Caracterização física, química e anatômica da madeira de Terminalia ivorensis. Colombo, Embrapa Florestas, 1999. 14p. (Circular Técnica, 31)

MOAT, A.G.; FOSTER, J.W. \& SPECTOR, M.P. Microbial physiology. 4.ed. New York, Wiley-Liss, 2002. 714p.
MONTEIRO, M.T. \& GAMA-RODRIGUES, E.F. Carbon, nitrogen, and microbial biomass activity in different litter structures of a natural forest. R. Bras. Ci. Solo, 28:819826, 2004.

PARKIN, T.B.; DORAN, J.W. \& FRANCO-VIZCAÍNO, E. Field and laboratory tests of soil respiration. In: DORAN, J.W. \& JONES, A., eds. Methods for assessing soil quality. Madison, Soil Science Society of America, 1996. p.231-245.

PEÑA, M.L.P.; MARQUES, R.; JAHNEL, M.C. \& ANJOS, A. Respiração microbiana como indicador da qualidade do solo em ecossistema florestal. Floresta, 35:117-127, 2005.

RIGOBELO, E.C. \& NAHAS, E. Flutuação sazonal da comunidade bacteriana e da atividade microbiana em solos sob eucalipto e pinus. Sci. Agric., 61:88-93, 2004.

SPSS. Sigmaplot 2006 for Windows, version 10.0. Chicago, 2006.

TAUK, S.M. Biodegradação de resíduos orgânicos no solo. R. Bras. Geoci., 20:299-301, 1990

TÓTOLA, M.R. \& CHAER, G.M. Microrganismos e processos microbiológicos como indicadores da qualidade dos solos. In: ALVAREZ V., V.H.; SCHAEFER, C.E.G.R.; BARROS, N.F.; MELLO, J.W.V. \& COSTA, L.M., eds.. Tópicos em ciência do solo. Viçosa, MG, Sociedade Brasileira de Ciência do Solos, 2002. v.2. p.195-275.

URBINATI, C.V.; AZEVEDO, A.A. \& SILVA, E.A. Variação estrutural quantitativa no lenho de Terminalia ivorensis A. Chev., Combretaceae. Acta Bot. Bras., 17:421-437, 2003. 